\title{
Ruteo de buses escolares con consideraciones ambientales mediante Búsqueda Tabú Granular
}

\author{
School bus routing problem with environmental considerations using granular tabu \\ search
}

\author{
P. A. Lázaro-González iD , J. E. Arias-Osorio iD ; J. P. Orejuela-Cabrera iD
}

\begin{abstract}
Resumen - En el contexto actual es importante que el ruteo de buses escolares (SBRP) además de la eficiencia aborde también las dimensiones social y ambiental, para garantizar soluciones sostenibles. La dimensión ambiental ha sido abordada ampliamente en el VRP, sin embargo, el SBRP no ha contado con la misma suerte, a pesar de que existen estudios sobre la relación entre los problemas ambientales en el trasporte escolar y salud de los niños, no obstante, no se encuentran trabajos que aborden la dimensión ambiental en el ruteo de buses escolares, en tal sentido este artículo aborda el SBRP con consideraciones ambientales. Se formula un modelo matemático que minimiza el consumo de combustible, que se calcula en función de la distancia recorrida, el peso de los vehículos y el de los estudiantes. El modelo es resuelto de manera óptima para instancias pequeñas, y para instancias de mayor tamaño se emplea un algoritmo basado en Búsqueda Tabú Granular. La solución inicial es generada por el algoritmo de ahorros. Se evalúa el rendimiento del algoritmo comparando los tiempos y valores de función objetivo con respecto al método exacto, la meta heurística generó soluciones $\mathbf{9 9 , 9 6 \%}$ en promedio más rápido que el método exacto y generó soluciones con valor de función objetivo alejado en promedio $13,98 \%$ de las del método exacto. En este trabajo se hace una extensión al SBRP, adicionando la dimensión ambiental, aproximado el consumo de combustible en función de la distancia y el peso.
\end{abstract}

Palabras claves - Búsqueda Tabú Granular; Green VRP; Ruteo de Vehículos Escolares.

Abstract - in the current context it is important that the School Bus Routing Problem (SBRP) in addition to efficiency also addresses the social and environmental dimensions, to ensure sustainable solutions. The environmental dimension has been widely addressed in the VRP, however, the SBRP has not had the same

Este manuscrito fue enviado el 19 de agosto de 2019 y aceptado el 20 de diciembre de 2019.

Este trabajo proyecto de investigación, contó con el apoyo conjunto de la Universidad Industrial de Santander y la Universidad del Valle. En el proceso de dirección y codirección de la investigación.

Paola A. Lázaro-González, Universidad Industrial de Santander, Escuela de Estudios Industriales y Empresariales, Ciudadela universitaria, Calle 9 \#\#27, Bucaramanga, Santander, paolalazaro@uis.edu.co development, although there are studies on the relationship between environmental problems in school transport and children's health, however, there are no jobs that address the environmental dimension in the SBRP, in this sense this article addresses the SBRP with environmental considerations. A mathematical model is formulated to minimize fuel consumption, which is calculated based on the distance traveled, the weight of the vehicles and the students. The model is optimally solved for small instances, and for larger instances an algorithm based on Granular Tabu Search is used. The initial solution is generated by the savings algorithm. The performance of the algorithm is evaluated by comparing the times and values of objective function with respect to the exact method, the times of solution with the metaheuristic were $99.96 \%$ on average faster than the exact method, however the objective values was far on average $13.98 \%$ of the exact method. In this work an extension to the SBRP is made, the environmental dimension is added, approximate the fuel consumption according to the distance and the weight.

Index Terms - Granular Taboo Search; Green VRP; School Bus Routing Problem;

\section{INTRODUCCIÓN}

El estudio del problema de rutas escolares inicia en los años 60 cuando Rita Newton y Warren Thomas realizan la primera publicación acerca del tema, en donde relatan que la planeación de rutas se realizaba manualmente utilizando un mapa de la ciudad y un listado de los datos,[1]. La evolución de tema de investigación ha sido importante y todavía se continúa con el desarrollo de nuevos métodos y técnicas que permiten dar soluciones a problemas cada vez más complejos ya que consideran mayores elementos que se ajustan a los nuevos requerimientos de la sociedad.

J. E. Arias-Osorio, Universidad Industrial de Santander, Escuela de Estudios Industriales y Empresariales, Ciudadela universitaria, Calle 9 \#27, Bucaramanga, Santander, jearias@uis.edu.co

J. P. Orejuela-Cabrera, Universidad del Valle, Escuela de Ingeniería Industrial, Ciudadela universitaria Meléndez, Cl. 13 \#100-00, Cali, Valle del Cauca, juan.orejuela@correounivalle.edu.co, Autor corresponsal. Universidad Nacional de Colombia, Facultad de ingenierías, Doctorado en ingeniería Industria y Organizaciones, jporejuelac@unal.edu.co 
Una de las consideraciones que ha tomado gran importancia en la sociedad y en las investigaciones en el problema de ruteo, es el tema ambiental [2]. La implementación de medidas que reducen de algún modo la contaminación ambiental por medio de características añadidas a modelos [3], ha sido una estrategia usada en el problema del ruteo de vehículos de carga. Sin embargo en el ruteo de buses escolares, el tema se ha abordado de manera general en el marco general del transporte escolar [4] o en su relación con el tema de la salud [4]. Sin embargo, no se encuentran publicaciones que aborden el tema ambiental en contexto del problema del ruteo de buses escolares.

En este orden de ideas, en la presente investigación se desarrolla un modelo matemático para ruteo de buses escolares que considera el aspecto ambiental, mediante la definición de una función de desempeño que minimiza el consumo de combustible, el cual se calcula en función de la distancia recorrida y el peso que mueve el vehículo cuando sale de cada nodo. El modelo se resuelve de manera óptima para instancias pequeñas y para instancias grandes se implementa un algoritmo de búsqueda tabú granular.

En las siguientes secciones el trabajo se ha organizado de la siguiente manera, en la sección II una revisión de la literatura alrededor del problema, en la sección III se presenta la descripción y formulación matemática del problema, en la sección IV se describe el enfoque de solución empleado, en la sección V se presentan los resultados para finalmente en la sección VI presentar las conclusiones.

\section{REVISIÓN DE LA LITERATURA}

El SBRP se encuentra clasificado en la literatura como un problema de optimización combinatoria [5], el cual busca diseñar rutas para una flota de buses escolares con el objetivo de minimizar el tiempo y los costos [6] al prestar el servicio de transporte de estudiantes hacia sus respectivas escuelas o colegios en áreas rurales o urbanas.

En el problema se identifican conjuntos de estudiantes, de escuelas, de calles, de vehículos y de paradas, y general el problema según las decisiones que se tomen puede ser dividido en cinco subproblemas, los cuales son: preparación de datos, selección de paradas, generación de rutas, ajuste de ventana de tiempo y horarios de rutas.

La preparación de datos: en esta etapa se definen los tipos de datos que se utilizarán, por ejemplo, la red de calles a utilizar, los vecindarios que se agruparán, los estudiantes que viven allí y los parámetros a usar.

La selección de paradas: es la elección de un conjunto de paradas ubicadas sobre la red de calles definidas anteriormente por medio de la aplicación de heurísticas, ya que este paso se encuentra clasificado como una estrategia de locationallocation-routing (LAR) o allocation-routing-location (ARL) para el problema desarrollado en áreas urbanas; puesto que, si el problema es desarrollado en áreas rurales las paradas serán la casa de los estudiantes y no será necesario hacer uso de métodos o técnicas para encontrarlas [7].

La generación de rutas de buses en la que se desarrolla el algoritmo que va definir las rutas que cumplen con las restricciones añadidas al problema. El Ajuste de ventana de tiempo que, una vez obtenidas las rutas óptimas, algunos problemas son estudiados con restricciones de tiempo los cuales son, la hora de apertura y la hora de salida de la escuela. La definición de horario de ruta: finalmente, se define la hora de partida de cada una de las rutas y la secuencia en la cual se debe realizar [8].

Al respecto, se encuentra que no es regla definir la investigación bajo los cinco pasos mencionados anteriormente, sino que de lo contrario algunos investigadores desarrollan el problema con su propia versión, la cual puede ser: dividir el problema solo en dos pasos, por ejemplo, preparación de datos y selección de paradas [9], pues depende del objetivo que se busca con la investigación. También, se puede ver en la literatura que el problema puede ser multiobjetivo o bi-objetivo, como es realizado en algunos casos en donde los objetivos son: minimizar el tiempo de servicio y al mismo tiempo, minimizar los costos operacionales según [10].

Así mismo, la solución de este tipo de problemas no tiene estipulado un método específico, sino que al contrario existe una gran variedad de ellos. Por un lado están las heurísticas y meta heurísticas que se puede revisar en [11]-[14], y por otro lado las técnicas exactas [15]-[17].

Por el lado de las meta heurísticas se encuentran investigaciones en ruteo de buses escolares que emplean búsqueda tabú [14], algoritmos genéticos [5], optimización por enjambre de partículas [18], colonia de hormigas [19], la meta heurística GRASP [20] entre otras. Sim embargo, no se encuentran investigaciones que empleen la búsqueda tabú granular en el problema del rute de buses escolares, a pesar de que ha gozado de gran aceptación en problema de ruteo de vehículos de carga [21].

Por otro lado, en cuanto al tema medio ambiental, desde lo público, en cuanto a Colombia respecta, se encuentra un marco normativo que propende por la creación de medidas que permitan la disminución de contaminación del aire, como por ejemplo "la Política de Prevención y Control de la contaminación del aire", la cual tiene como objetivo general: "impulsar la gestión de la calidad del aire en el corto, mediano y largo plazo, con el fin de alcanzar los niveles de calidad del aire adecuados para proteger la salud y el bienestar humano en el marco del desarrollo sostenible" [22].

La problemática ambiental asociada al transporte está en la agenda del gobierno, ya que las cifras implican la definición de estrategias. De manera específica el sector transporte generó 22 millones de toneladas de $\mathrm{CO} 2$ de las emisiones totales del país, lo que representó un $10 \%$ en el año 2010 y se proyecta una emisión de 9,72 millones de toneladas en 2030, las cuales serán un $20 \%$ de las emisiones proyectadas por el uso de automotores [23]. 
Por otro lado, en [24], se hace un presentación sobre los combustibles limpios y límites máximos de emisión en prueba dinámica para vehículos de prestación de servicio de transporte de pasajeros, en lo que atañe al tema de investigación considera "que es necesario establecer niveles máximos de emisión permisibles para fuentes móviles que se vinculen a la prestación de servicio de transporte público de pasajeros que operen con combustibles limpios.

El Gobierno Colombiano ha definido como estrategias para promover la reducción de emisiones por parte de los vehículos las siguientes: La mejorara la calidad de los combustibles. El ingreso de tecnologías más limpias. El fortalecimiento del mantenimiento vehicular. La renovación del parque automotor y la implementación de sistemas sostenibles [23]. Y orientados a esta última estrategia es donde se puede circunscribir trabajo como el presente, en el que se considera lo ambiental, buscado minimizar el consumo de combustible.

\section{DEFINICIÓN DEL PROBLEMA}

El SBRP puede ser descrito como un problema de optimización de rutas que busca minimizar el consumo de combustible total, considerando a $V=\{0,1,2, \ldots i\}$ como el conjunto de paradas $(0$ corresponde a la escuela), a $K=\{1,2, \ldots k\}$ como el conjunto de vehículos y a $E=\{12,13, \ldots i j\}$ como el conjunto de arcos, $C_{k}$ como la capacidad del vehículo $k, d_{i}$ cantidad de niños a recoger en el nodo $i, p_{i}$ peso del total de niños a recoger en el nodo $i, S_{I}$ el tiempo de espera en el nodo $i, d i s_{i j}$ la distancia del nodo $i$ al nodo $j$, $W v e h_{k}$ peso del autobús $k$ vacío. El modelo matemático formulado resulta con la siguiente estructura:

$$
\begin{aligned}
& \operatorname{Min} z=\sum_{k \in K} a 1 * \text { Vveh }_{k} * V e h_{k}+\sum_{j \in V} a 1 * P E_{j} \\
& \text { * } X_{i j k} \\
& \sum_{j \in V} X_{i j k}-\sum_{j \in V} X_{j i k}=0 \quad \forall i \in V, \quad \forall k \in K \\
& \sum_{j \in V} X_{j i k}-Y_{i k}=0 \quad \forall i \in V, \quad \forall k \in K \\
& \sum_{j \in V} X_{i j k}-Y_{i k}=0 \quad \forall i \in V, \quad \forall k \in K \\
& |V| Y_{0 k}-\sum_{i \in V / i \neq 0} Y_{i k} \geq 0 \quad \forall k \in K \\
& \sum_{k \in K} Y_{i k}=1 \quad \forall i \in V \backslash\{0\}
\end{aligned}
$$$$
+\sum_{i \in V} \sum_{j \in V} \sum_{k \in K} a 2 * d i s_{i j} * X_{i j k}+\sum_{i \in V} \sum_{j \in V} \sum_{k \in K} b
$$

$$
\begin{aligned}
& F_{i}+d_{i} \leq F_{j}+M *\left(1-\sum_{k \in K} X_{i j k}\right) \\
& \forall i, \in V \quad i \neq j, \quad j \neq 0 \\
& M *\left(1-\sum_{k \in K} X_{i j k}\right)+F_{i}+d_{i} \geq F_{j} \\
& \forall i, j \in V \quad i \neq j, \quad j \neq 0 \\
& P E_{i}+p_{i} \leq P E_{j}+N *\left(1-\sum_{k \in K} X_{i j k}\right) \\
& \forall i, j \in V \quad i \neq j, \quad j \neq 0 \\
& N *\left(1-\sum_{k \in K} X_{i j k}\right)+P E_{i}+p_{i} \geq P E_{j} \\
& \forall i, j \in V \quad i \neq j, \quad j \neq 0 \\
& T E_{i}+\sum_{k \in K} X_{i j k} *\left(\frac{d i s_{i j}}{v_{k}}+s_{i}\right) \leq T E_{j}+T *\left(1-\sum_{k \in K} X_{i j k}\right) \\
& \forall i, j \in V \quad i \neq j, \quad j \neq 0 \\
& T *\left(1-\sum_{k \in K} X_{i j k}\right)+T E_{i}+\sum_{k \in K} X_{i j k} *\left(\frac{d i s_{i j}}{v_{k}}+s_{i}\right) \geq T E_{j} \\
& \forall i, j \in V \quad i \neq j, \quad j \neq 0 \\
& \sum_{i \in V} Y_{i k} * d_{i} \leq C_{k} \quad \forall k \in K \\
& Q * V e h_{k} \geq \sum_{i \in V} Y_{i k} \quad \forall k \in K
\end{aligned}
$$

Donde $X_{i j k}$ es una variable binaria de decisión que será uno si el autobús $k$ atraviesa el arco $i j, Y_{i k}$ será uno si el autobús $k$ visita la parada $i$ y $V e h_{k}$ será uno si el autobús $k$ se habilita para la ruta, $F_{i}$ es la cantidad de niños que lleva el vehículo cuanto llega al nodo $i, P E_{i}$ es el peso total de los niños que lleva el vehículo cuando llega al nodo $i$ y $T E_{i}$ tiempo de entrada del vehículo al nodo $i$.

La función objetivo (1) minimiza el consumo de combustible total en función del peso y la distancia recorrida. Las restricciones: (2) garantiza que si un vehículo $k$ entra al nodo $i$ salga de este; (3) asegura que la sumatoria de todas las aristas que salen del nodo $i$ en el vehículo $k$ sean igual a 1 , si la parada es visitada; (4) asegura que la sumatoria de todas las aristas que llegan al nodo $i$ en el vehículo $k$ sean igual a 1, si la parada es visitada; (5) asegura que todos los vehículos habilitados inicien su recorrido en la escuela; (6) asegura que cada parada no sea visitada más de una vez a excepción de la escuela.

Las restricciones (7) y (8) aseguran que los niños que un vehículo lleve al llegar al nodo $j$ sea igual a la suma de los niños 
que llevaba al llegar a $i$ y los que recogió en $i$; (9) y (10) aseguran que el peso de los niños que un vehículo lleve al llegar al nodo $j$ sea igual a la suma del peso de los niños que llevaba al llegar a $i$ y el peso de los que recogió en $i$; (11) y (12) asegura que el tiempo de ruta que un vehículo lleva al llegar al nodo $j$ sea igual a la suma del tiempo de ruta que llevaba al llegar a $i$, el tiempo de espera en el nodo $i$ y el tiempo que demora en recorrer el arco $i j$; (13) asegura que no se supere la capacidad máxima del vehículo $k$; (14) indica que vehículo se habilita; (15) y (16) asegura que la variable $X$ y la variable $Y$ sean binarias.

La función objetivo fue propuesta tomando como base tomada de [25] y fue adaptada a las variables utilizadas en el modelo, los coeficientes $a_{1}=4,0864, a 2=4,8771, \quad$ y $b=-14,3004$, fueron estimados mediante el mismo procedimiento mencionado en [25], con datos reales suministrados por una empresa de transporte escolar de la ciudad de Bucaramanga.

\section{TÉCNICAS DE SOLUCIÓN}

En la solución del problema se implementa como solución inicial el Algoritmo de ahorros de Clarke and Wright [26], para posteriormente partir de dicha solución en el algoritmo desarrollado con base en la meta heurística Búsqueda Tabú Granular [21].

\section{A. Algoritmo de Ahorros Clarke and Wright}

La solución inicial se basa en el Algoritmo de ahorros, puesto que esta manera se obtiene una solución en tiempo computacional reducido. A continuación, se observa en detalle el algoritmo.

\section{Algoritmo 1}

Entrada: vector paradas, matriz distancias, vector estudiantes asignados, vector capacidad de vehículos, vector peso vehículos y coeficientes de la regresión.

Salida: rutas iniciales. Inicio

1. Crear rutas individuales.

2. Calcular estudiantes a recoger por ruta.

3. Calcular distancia por ruta.

4. Asignar vehículo a cada ruta.

5. Calcula matriz de ahorros. Si No existen más ahorros FIN.

6. Encontrar coordenadas del mayor ahorro.

7. Calcular el número de estudiantes a recoger en el arco encontrado en (7).

8. Asignar un vehículo a la ruta creada.

9. Si el número de estudiantes es menor a la capacidad de la ruta. Crear la ruta.

10. Eliminar los ahorros de la columna y la fila.

11. Eliminar la ruta individual para el nodo $j$ de la ruta.

12. Si existe un ahorro mayor saliendo de $j$ asignar el mayor ahorro a la ruta. Si No FIN DE LA RUTA.

13. Calcular el total de estudiantes a recoger.
14. Si el total de estudiantes es menor o igual a la capacidad del vehículo asignado vaya al paso $10 \mathrm{y}$ repita. Si No FIN DE LA RUTA.

15. Finalizar la ruta en la escuela.

16. Asignar el total de estudiantes a la ruta.

17. Calcular distancia total.

18. Calcula el consume de combustible total.

\section{Fin}

La asignación de vehículos se realiza de menor a mayor capacidad y el algoritmo en algunos casos permite soluciones infactibles en cuanto a total de vehículos, es decir, tiene permitido tomar el vehículo dos las veces que sea necesario si el no hay más vehículos disponibles.

\section{B. Meta heurística Búsqueda Tabú Granular}

La meta heurística parte de la solución inicial generada por el algoritmo mencionado en (3.1). El algoritmo desarrollado se basa en [21] y [27], a continuación, se observa en detalle el algoritmo.

\section{Algoritmo 2}

Entrada: vector paradas, matriz distancias, vector estudiantes asignados, vector capacidad de vehículos, vector peso vehículos, coeficientes de la regresión y solución inicial.

Salida: rutas finales.

Inicio

19. Calcular umbral de granularidad $\vartheta$.

20. Generar intercambio posible i y j.

21. Si i es diferente a $\mathrm{j}$ calcular la distancia entre nodos.

22. Si No repetir paso dos y tres.

23. Si la distancia es menor a $\vartheta$ intercambiar los nodos.

24. Si No volver al paso dos y repetir.

25. Guardar la nueva solución como Xsalida.

26. Guardar Xsalida como aux.

27. Si Aux tiene en la primera posición un 1, volver al paso dos y repetir.

28. Si No calcular la capacidad de cada una de las rutas.

29. Cada una de las capacidades guárdela como Cap1.

30. Asignar un vehículo a la ruta.

31. Si Cap1 es menor o igual a la capacidad del vehículo asignado, asignar Cap1 como el número de estudiantes de la ruta.

32. Si No volver a 12 y repetir.

33. Guardar la ruta.

34. Guardar el número del vehículo asignado.

35. Guardar el número de estudiantes.

36. Calcular el consumo de combustible y guardar.

37. Guardar la solución completa.

38. Guardar el arco que se intercambió.

39. Si Cap1 es diferente de 0 , sacar el vehículo asignado dentro de los posibles.

40. Si No, Si Cap1 es igual a 0, dejar el vehículo dentro de los disponibles.

41. Si No sumar el consumo total de todas las rutas de la solución. 
42. Si es la última ruta de la solución, guardar el consumo total de dicha solución.

43. Si No, Si Cap1 es mayor que la capacidad máxima de los vehículos, asignar penalización al consumo total de la ruta.

44. Si No, volver Consumo total de combustible igual a Consumo mínimo.

45. Encontrar los nodos del intercambio.

46. Si los arcos No se encuentran en la Lista tabú. Solución Tabú=Xsalida_inicial y Consumo mínimo=Consumo total de la solución.

47. Si se encuentran los arcos en la Lista Tabú, verificar si el Consumo mínimo es menor a Z. Si No volver al paso uno y repetir.

48. Si se encuentra en la Lista Tabú, guardar Solución Tabú como Xsalida_inicial.

49. Si No Guardar en la Lista Tabú y guardar Solución Tabú como Xsalida_inicial.

50. Guardar Consumo mínimo como Z.

51. Guardar Consumo mínimo como Consumo total de la solución.

52. Si el número de iteraciones es igual a 10, volver B1 en B2.

53. Si No, Si el número de iteraciones es igual a 15 , volver B1 en B1.

54. Si No ir al paso uno y repetir.

55. Parar después de 20 iteraciones.

\section{Fin}

La meta heurística tabú granular comparte las mismas características de la búsqueda tabú con la única diferencia de que no utiliza el grafo completo de solución, sino que utiliza aquellos elementos de la población inicial que son incidentes a la escuela y todas las combinaciones de los arcos pertenecientes a la solución inicial que tengan una distancia menor al umbral de granularidad $\vartheta$, dado por la siguiente ecuación:

$$
\vartheta=\beta * \frac{z^{\prime}}{(n+K)}
$$

Donde $\beta$ es el parámetro de dispersión positivo, $z^{\prime}$ es el valor objetivo de la solución inicial $f_{1}\left(S_{o}\right), n$ es el número de estudiantes y $K$ es el número de rutas obtenidas en la solución inicial.

La meta heurística puede aplicar como criterio de aspiración el hecho de que si el valor de la función objetivo de la nueva solución es menor $f_{2}\left(S_{o}\right)<f_{1}\left(S_{o}\right)$, el movimiento que produce a $\mathrm{S}$ es ejecutado inclusive si este es un movimiento tabú. También, las estrategias de diversificación e intensificación se vinculan a los valores de dispersión $\beta$, de manera que $\beta$ tomará valores grandes para encontrar soluciones no visitadas antes (inducir procesos de diversificación) y $\beta$ tomará valores pequeños para encontrar soluciones cercanas a la mejor solución encontrada (permitir procesos de intensificación) [5].

La restricción que lleva a pensar en un vecindario granular es: "generalmente el VRP es definido en un grafo completo y se puede observar que los arcos largos tienen poca probabilidad de ser parte de buenas soluciones" [14]. Esto se debe a que los arcos largos tienen asociado un costo alto (en el caso del problema de ruteo de vehículos) y por ende, al buscar minimizar costos o distancia recorrida estos no serán tomados en consideración para una buena solución, como se puede observar en el artículo [14]. También mencionan estos autores que los tiempos de ejecución disminuyen.

Otro punto vinculado al vecindario granular es que la modificación de la estructura del grafo bajo la condición de granularidad permite la inclusión de estrategias de intensificación y diversificación, modificando el valor del parámetro $\beta$, puesto que lo que hace es que altera el grafo y nuevas soluciones pueden ser encontradas.

La solución inicial ingresa como un vector que contiene todas las rutas y el valor de la función objetivo de dicha solución ingresa con el nombre de $Z$. Los valores de los parámetros del algoritmo dos tomaron los siguientes valores:

TABLA I

\begin{tabular}{ll}
\multicolumn{2}{c}{ VALORES DE LOS PARÁMETROS } \\
\hline Parámetro & Valor Obtenido \\
\hline \hline $\boldsymbol{B 1}$ & 1.5 \\
$\boldsymbol{B} 2$ & 4.5 \\
N_change & 10 \\
\hline \hline
\end{tabular}

El valor de N_change se aplica en la operación 32 del diagrama de flujo y para la operación 33 se aplica el N_vuelta con un valor de 15, el cual fue definido por pruebas realizadas al algoritmo desarrollado para esta investigación.

En el algoritmo se aplicaron una serie de características de Búsqueda Tabú, las cuales son:

Criterio de aspiración: esta estrategia se aplica al aceptar movimientos así sean un movimiento tabú, solo sí este intercambio genera un consumo de combustible menor a la mejor solución encontrada durante la búsqueda.

Estrategia de diversificación: esta estrategia se aplica tomando valores grandes de $B$ (en este caso 4.5) con el fin de explorar soluciones no visitadas anteriormente.

Estrategia de intensificación: esta estrategia se aplica tomando valores pequeños de $B$ (en este caso 1.5 ) con el fin de explorar soluciones cercanas a la mejor encontrada anteriormente.

Tipo de movimiento: el tipo de movimiento aplicado en el algoritmo fue el movimiento de inserción.

Tenure: tiene un valor de 5 .

Lista tabú: la lista tiene un tamaño de 5.

Otras estrategias utilizadas en el desarrollo del algoritmo fueron:

Penalización: este valor de penalización, el cual es igual a la gran $\mathrm{M}$, se aplica con el objetivo de diferenciar las soluciones que contienen rutas que superan la capacidad máxima del vehículo con mayor capacidad y evita que sea tomado como consumo mínimo por el algoritmo. 
Rutas con dos unos seguidos: esto quiere decir que hay menos rutas de las que contaba la solución inicial, por ejemplo, Solución inicial $=\left[\begin{array}{llllllll}1 & 2 & 3 & 4 & 1 & 5 & 6171\end{array}\right]$ tiene 3 rutas y se resultan dos unos seguidos podría resultar así

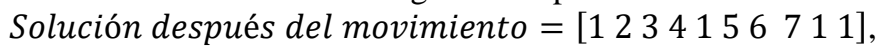
como se puede observar se redujeron las rutas a 2 . A lo anterior el algoritmo lo identifica cuando la variable Capl toma el valor de 0 y este asigna a la ruta 11 un valor de 0 como función objetivo y no le asigna vehículo. Esto evita que se tomen vehículos por dicha ruta y se generen consumos inexistentes.

\section{RESULTADOS COMPUTACIONALES}

Esta etapa de la investigación se llevó a cabo mediante el uso del software Sistema General de Modelaje Algebraico (GAMS) el cual permite modelar problemas de optimización lineales, no lineales o mixtos mediante el método exacto de ramificación y acotamiento. También, se hace uso del software de programación Laboratorio de matrices (MATLAB), el cual permite la implementación de la técnica heurística y meta heurística aplicada en el presente trabajo.

Las instancias fueron creadas para el problema en particular, ya que el modelo resulto de estructura diferente a los encontrados en la literatura. Para claridad de los resultados en la tabla II se encuentran las características de la flota de vehículos utilizada.

TABLA II.

CARACTERÍSTICAS DE LA FLOTA DE VEHÍCULOS

\begin{tabular}{llll}
\hline \hline Tipo de vehículo & Marca & $\begin{array}{l}\text { Capacidad } \\
\text { (Pasajeros) }\end{array}$ & $\begin{array}{l}\text { Peso } \\
\text { vehículo } \\
\text { vacío (ton) }\end{array}$ \\
\hline \multirow{4}{*}{ Micro buses } & Hyundai & 12 & 2,265 \\
& Volkswagen & 16 & 2,883 \\
& Nissan Urvan & 16 & 1,920 \\
& Renault & 18 & 2,007 \\
Busetas & Kia & 19 & 2,207 \\
& Nissan & 23 & 2,340 \\
Buses & Hyundai & 23 & 4,185 \\
& Chevrolet & 23 & 5,200 \\
& Chevrolet & 37 & 6,407 \\
\hline \hline
\end{tabular}

Nota: Datos suministrados por la empresa de transporte.

\section{A. Método exacto vs Meta heurística}

El tiempo de ejecución de la meta heurística es el total entre el tiempo de ejecución del algoritmo de ahorros y el tiempo del algoritmo Búsqueda Tabú Granular, ya que el último necesita del primero para encontrar soluciones. A continuación, los resultados.

TABLA III

MÉTODO EXACTO VS META HEURÍSTICA

\begin{tabular}{lllllll}
\hline \hline \multirow{2}{*}{ Inst } & \multicolumn{2}{c}{ Exacto } & \multicolumn{2}{c}{ Metaheur. } & \multicolumn{2}{l}{ Diferencia } \\
\cline { 2 - 7 } & $\mathrm{t}$ & $\mathrm{FO}$ & $\mathrm{t}$ & $\mathrm{FO}$ & $\Delta \mathrm{t}$ & $\Delta \mathrm{FO}$ \\
\hline Ins1 & 0,17 & 7.775 & 0,06 & 9184 & $63,5 \%$ & $18,1 \%$ \\
Ins2 & 0,33 & 7.720 & 0,06 & 9133 & $80,8 \%$ & $18,3 \%$ \\
Ins3 & 2,92 & 9.425 & 0,07 & 20906 & $97,4 \%$ & $121 \%$ \\
Ins4 & 19,5 & 9.368 & 0,07 & 20844 & $99,6 \%$ & $122 \%$
\end{tabular}

\begin{tabular}{lllllll} 
Ins5 & 25,8 & 17.507 & 0,08 & 28609 & $99,7 \%$ & $63,4 \%$ \\
Ins6 & 21,9 & 17.530 & 0,07 & 28968 & $99,7 \%$ & $65,2 \%$ \\
Ins7 & 12,84 & 17.505 & 0,08 & 28120 & $99,4 \%$ & $60,6 \%$ \\
Ins8 & 52,09 & 25.245 & 0,18 & 36717 & $99,7 \%$ & $45,4 \%$ \\
Ins9 & 76,63 & 25.129 & 0,10 & 46182 & $99,9 \%$ & $83,9 \%$ \\
Ins10 & 999,6 & 104140 & 0,10 & 36387 & $99,9 \%$ & $-65 \%$ \\
Ins11 & 999,6 & 129832 & 0,11 & 36371 & $99,9 \%$ & $-71 \%$ \\
Ins12 & 898,4 & 35006 & 0,08 & 36354 & $99,9 \%$ & $3,85 \%$ \\
Ins13 & - & & 0,11 & 45293 & - & \\
Ins14 & - & & 0,10 & 45153 & - & \\
Ins15 & - & & 0,11 & 70995 & - & \\
Ins16 & - & & 0,10 & 54627 & - & \\
Ins17 & - & & 0,10 & 54551 & - & \\
Ins18 & - & & 0,11 & 54493 & - & \\
Ins19 & - & & 0,10 & 54380 & - & \\
Ins20 & - & & 0,11 & 54348 & - & \\
\hline \hline Prom & 259,15 & 33.848 & 0,09 & 38581 & $99,9 \%$ & $13,9 \%$ \\
\hline \hline
\end{tabular}

La tabla III contiene la diferencia de tiempos y valor de función objetivo para las instancias de 1 a 12, puesto que como se mencionó anteriormente el método exacto no encontró soluciones para las demás instancias, ya que superó el tiempo límite de búsqueda de una solución. Los valores que se observan reflejan que en cuanto a tiempos de ejecución resulta la meta heurística más eficaz, pues generó soluciones 99,96\% en promedio más rápido que el método exacto. Con respecto a la función objetivo se encuentra que la meta heurística generó soluciones con valor de función objetivo 13,98\% mayor que el método exacto.

\section{CONCLUSIONES}

Se encuentra en la revisión de literatura un incremento de la conciencia ambiental a partir del año 2017, puesto que del total de los artículos revisados acerca de SBRP con consideraciones ambientales el $48 \%$ de ellos fueron publicados entre los años 2017 y comienzos del 2018, que fue cuando se realizó la revisión de literatura. A demás, se puede observar que los países que mayor aporte hicieron fueron China y USA con el $28 \%$ y $24 \%$ de las publicaciones.

En el método exacto se observa que las restricciones con estructura como las ecuaciones (7), (8), (9), (10), (11) y (12) crea el resultado esperado que es la cancelación de sub-tours y generan control sobre los resultados del problema, por lo que se considera una buena estrategia para la estructura de algunas de las restricciones de problemas de diseño de rutas.

También se observa, que la función objetivo (ecuación 1) aplicada no describe en detalle el comportamiento del consumo de combustible, pues este puede depender de otros factores, pero permite un acercamiento y estudio al detalle de la relación del consumo de combustible con factores más globales, como lo son en este caso: el peso del vehículo, el peso de los estudiantes, y la distancia recorrida, lo cual lleva a la conclusión 
de que el factor que más influye sobre el consumo es el tipo de vehículo, en específico el peso del vehículo vacío.

En cuanto al diseño del algoritmo de la meta heurística Búsqueda Tabú Granular que la estrategia de asignación de vehículos de menor a mayor capacidad aplicada no funciona, para problemas que consideran la disminución del consumo de combustible, puesto que el factor que mayor impacto tiene sobre este es el peso del vehículo.

Búsqueda Tabú Granular es una meta heurística de alta calidad en la aplicación de problemas de diseño de rutas con función objetivo de minimización de costos o de distancia recorrida. En cuanto a función objetivo de minimización de consumo de combustible con flota de vehículos heterogénea no funciona igual, sin embargo se observa que para las instancias que se pudieron comparar con el método exacto, en dos de ellas el algoritmo generó soluciones con un valor de función objetivo un $18 \%$ más alto y en una de ellas solo un $3,85 \%$ más alto que el método exacto, pero en promedio un $13,98 \%$ más alto con tiempos computacionales $99,96 \%$ en promedio menores que el método exacto, lo cual la sigue haciendo el algoritmo desarrollado eficiente frente a los métodos exactos.

Se propone como investigación futura incluir en el problema la selección de tipo de flota de tal modo que se pueda estudiar la influencia del tipo de combustible y tipo el motor en el consumo, esto de cara a la entrada de los vehículos eléctricos.

Se propone como investigación futura relacionar el problema de rutes de buses escolares con consideraciones ambientales con los impactos en la salud de dichas emisiones.

\section{REFERENCIAS}

[1] R. M. Newton and W. H. Thomas, "Design of school bus routes by computer,” Socioecon. Plann. Sci., vol. 3, no. 1, pp. 75-85, 1969, DOI: 10.1016/0038-0121(69)90051-2.

[2] C. Lin, K. L. Choy, G. T. S. Ho, S. H. Chung, and H. Y. Lam, "Survey of Green Vehicle Routing Problem: Past and future trends," Expert Syst. Appl., vol. 41, no. 4 PART 1, pp. 1118-1138, Mar. 2014, DOI: 10.1016/j.eswa.2013.07.107.

[3] T. Kramberger, K. Prah, and D. Dragan, "A heuristic approach to reduce carbon dioxide emissions," Proc. ICE - Transp., vol. 167, no. 5, pp. 296-305, Oct. 2014, DOI: 10.1680/tran.11.00053.

[4] T. Beatty and J. Shimshack, "School buses, diesel emissions, and respiratory health," J. Health Econ., vol. 30, no. 5, pp. 987-999, 2011, DOI: 10.1016/j.jhealeco.2011.05.017.

[5] S. A. Oluwadare, I. P. Oguntuyi, and J. C. Nwaiwu, "Solving school bus routing problem using genetic algorithm-based model," Int. J. Intell. Syst. Appl., vol. 10, no. March, pp. 50-58, 2018, DOI: 10.5815/ijisa.2018.03.06.

[6] A. Corberan et al., "Heuristic solutions to the problem of routing school buses with multiple objectives," J. Oper. Res. Soc., vol. 53, no. 4, pp. 427-435, 2002, DOI: 10.1057/palgrave.jors.2601324.

[7] G. Laporte, "Location-Routing Problems," in Location-routing problems., North-Holland, 1988, pp. 163-198.

[8] J. Park and B.-I. Kim, "The school bus routing problem: A review," Eur. J. Oper. Res., vol. 202, no. 2, pp. 311-319, Apr. 2010, DOI: 10.1016/j.ejor.2009.05.017.

[9] J. F. Sarubbi, C. M. Mesquita, E. Wanner, V. F. Santos, and C. M. Silva, "A strategy for clustering students minimizing the number of bus stops for solving the school bus routing problem," in Proceedings of the NOMS 2016 - 2016 IEEE/IFIP Network Operations and
Management Symposium, 2016, pp. 1175-1180, DOI: 10.1109/NOMS.2016.7502983.

[10] J. Pacheco, R. Caballero, M. Laguna, and J. Molina, "Bi-Objective Bus Routing: An Application to School Buses in Rural Areas," Transp. Sci., vol. 47, no. 3, pp. 397-411, Aug. 2013, DOI: $10.1287 /$ trsc. 1120.0437 .

[11] J. A. Addor, S. K. Amponsah, J. Annan, and C. Sebil, "School Bus Routing A Case Study of Wood Bridge School Complex, SekondiTakoradi, Ghana," Int. J. Bus. Soc. Res., vol. 3, no. 12, pp. 26-36, 2013, DOI: 10.18533/ijbsr.v3i12.346.

[12] J. Zhang and Y. Li, "School Bus Problem and its Algorithm," IERI Procedia, vol. 2, pp. 8-11, 2012, DOI: 10.1016/j.ieri.2012.06.043.

[13] B. Minocha and S. Triphati, "Solving School Bus Routing Problem Using Hybrid Genetic Algorithm: A Case Study," in Proceedings of the Second International Conference on Soft Computing for Problem Solving ( SocProS 2012 ), vol. 236, no. SocProS, B. V Babu, A. Nagar, J. Bansal, M. Pant, K. Deep, and K. Ray, Eds. New Delhi: Springer Verlag, 2014, pp. 93-103.

[14] P. Schittekat, J. Kinable, K. Sörensen, M. Sevaux, F. Spieksma, and J. Springael, "A metaheuristic for the school bus routing problem with bus stop selection," Eur. J. Oper. Res., vol. 229, no. 2, pp. 518528, Sep. 2013, DOI: 10.1016/j.ejor.2013.02.025.

[15] T. Bektaş and S. Elmastaş, "Solving school bus routing problems through integer programming," J. Oper. Res. Soc., vol. 58, no. 12, pp. 1599-1604, Dec. 2007, DOI: 10.1057/palgrave.jors.2602305.

[16] J. Kinable, F. C. R. Spieksma, and G. Vanden Berghe, "School bus routing-a column generation approach," Int. Trans. Oper. Res., vol. 21, no. 3, pp. 453-478, May 2014, DOI: 10.1111/itor.12080.

[17] Y.-K. Lee, S.-J. Jeong, H.-Y. Yun, and K.-S. Kim, "School Bus Routing Problem with Mixed-Load and Dynamic Arrivals," J. Korea Soc. Simul., vol. 22, no. 1, pp. 63-75, 2013, doi: 10.9709/jkss.2013.22.1.063.

[18] F. Su, Y. Li, S. Fulin, and L. Yueguang, "An improved quantumbehaved particle swarm algorithm and its application in school bus problem," in Proceedings - 2012 3rd International Conference on Digital Manufacturing and Automation, ICDMA 2012, 2012, pp. 198-201, DOI: 10.1109/ICDMA.2012.48.

[19] N.-A. Mokhtari and V. Ghezavati, "Integration of efficient multiobjective ant-colony and a heuristic method to solve a novel multiobjective mixed load school bus routing model," Appl. Soft Comput. J., vol. 68, pp. 92-109, 2018, DOI: 10.1016/j.asoc.2018.03.049.

[20] P. Schittekat, K. Sörensen, M. Sevaux, and J. Springael, "A metaheuristic for solving large instances of the school bus routing problem," in Proceedings of the 22nd European Conference on Operational Research (EURO '07), 2007, p. 2.

[21] P. Toth and D. Vigo, "The granular tabu search and its application to the vehicle-routing problem," INFORMS J. Comput., vol. 15, no. 4, pp. 333-346, 2004, DOI: 10.1287/ijoc.15.4.333.24890.

[22] Ministerio de Ambiente Vivienda y Desarrollo Territorial, "Política de Prevención y Control de la Contaminación del Aire," Ministerio de Ambiente, Vivienda y Desarrollo Territorial, no. 189. Bogotá D.C., p. 48, 2010.

[23] Ministerio de Ambiente y Desarrollo Sostenible, "Con movilidad sostenible Colombia podrá disminuir la contaminación del aire." Bogotá D.C. Colombia, pp. 5-6, 2017.

[24] V. Y. D. T. R. de C. Ministerio de Minas y Energía, de la Protección Social y de Ambiente, "RESOLUCIÓN NÚMERO 2604, 24 DIC 2009.” Bogotá D.C. Colombia, p. 29, 2009.

[25] İ. Küçükoğlu, S. Ene, A. Aksoy, and N. Öztürk, "A Green Capacitated Vehicle Routing Problem with Fuel Consumption Optimization Model," Int. J. Comput. Eng. Res., vol. 3, no. 7, pp. 1623, 2013.

[26] G. Clarke and J. W. Wright, "Scheduling of Vehicles from a Central Depot to a Number of Delivery Points Author," Oper. Res., vol. 12, no. 4, pp. 568-581, 1964, DOI: 10.1287/opre.12.4.568.

[27] D. Puenayán, J. Londoño, J. Escobar, and R. Linfati, "Un algoritmo basado en búsqueda tabú granular para la solución de un problema de ruteo de vehículos considerando flota heterogenea," Rev. Ing. Univ. Medellín, vol. 13, no. 25, pp. 284-294, 2014, doi: 10.22395/rium.v13n25a6. 
P. A. Lázaro-González was born in Bucaramanga, Santander, Colombia. She received the degree in industrial engineering from the Industrial University of Santander UIS., Colombia, in 2019. She is member of the playful application group as a teaching learning strategy GALEA from the UIS.

J. E. Arias-Osorio is Associate Professor of the Industrial University of Santander UIS. He is Industrial University of Santander UIS systems engineer with a master's degree in administration (with emphasis on electronic commerce). Professor in the area of quantitative methods (statistics and operations research). Member of the OPALO research group of the UIS (development of mathematical optimization models to solve problems in hospital logistics lines, design of distribution networks and urban distribution of goods).

J. P. Orejuela-Cabrera is Full professor of the school of Industrial Engineering at Universidad del Valle (Cali, Colombia). He has a Master degree in industrial engineering from and Industrial Engineer, from the Universidad del Valle. His current research interests: Applied mathematical modeling. Supply chain management and Simulation of production and logistics systems. He is member of the Research Group: Logistic y production from the Universidad del Valle. 